\title{
Prediction of Stock Market in Nigeria Using Artificial Neural Network
}

\author{
Peter Adebayo Idowu, Chris Osakwe, Aderonke Anthonia Kayode, Emmanuel Rotimi Adagunodo \\ Dept. of Computer Science and Engineering, Obafemi Awolowo University, Ile-Ife, Nigeria \\ paidowu@oauife.edu.ng; ned4christ@yahoo.com; omotonia@yahoo.com; eadagun@oauife.edu.ng
}

\begin{abstract}
Prediction of Nigerian stock market is almost not done by any researcher and is an important factor which can be used to determine the viability of Nigerian stock market. In this paper, the prediction models were developed using Artificial Neural Network. The result of the prediction of Nigerian Stock Exchange (NSE) market index value of selected banks using Artificial Neural Network was presented. The multilayer feed forward neural network was used, so that each output unit is told what its desired response to input signals ought to be. This work has confirmed the fact that artificial neural network can be used to predict future stock prices. The data collection period is from 2003 to 2006.
\end{abstract}

Index Terms - Artificial Neural Network, Prediction, Nigerian Stock Exchange, Input Signal

\section{Introduction}

Particle Prediction in finance especially in stock market is an important issue. Artificial Neural Network these days are being used to predict in financial sector. Artificial neural networks are parallel distributed information processing models that can recognize highly complex patterns within available data. . It is an information processing system that has certain performance characteristics in common with biological neural networks and therefore, each network is a collection of neurons that are arranged in specific formations. The basic elements of neural network comprise neurons and their connection strengths (weights). ANNs have been found to have a sound theoretical basis from the perspective of statistical learning theory as well as machine learning, and usually yield good performance when used for real-world data analysis[1] . Apart from that, ANNs have good generalization capabilities and are usually robust against noisy or missing data. All these and more make ANN highly desirable in the prediction of the Stock Market.

A Stock Market may be defined as a market where shares, stocks, government bonds, debentures, and other approved securities are sold and bought [2]. It is, thus, a market where large and small investors alike buy and sell through stock brokers, the stocks (or shares) of companies and government agencies. The idea of stock market prediction is not new; of course, business people often attempt to anticipate the market by interpreting external parameters, such as economic indicators, public opinion, and current political climate. Before the age of computers, people traded stocks and commodities primarily on intuition. As the level of investing and trading grew, people searched for tools and methods that would increase their gains while minimizing their risk.

The Nigerian stock market is by law principally managed and controlled by the Nigerian Stock Exchange which was established in 1960 through the Acts of Parliament and started operations in 1961 with 19 securities listed for trading. Today, there are more than 260 securities listed on the Exchange. Most of the listed companies have multinational affiliations and represent a cross-section of the economy, ranging from agriculture through manufacturing to services. The public trust in the NSE has grown tremendously, with about 3 million individual investors and $100 \mathrm{~s}$ of institutional investors, using the facilities of the Exchange.

A major challenge posed at any stock investor is the ability to predict stock prices; this is a great concern to both institutional and individual investors. Modeling techniques such as ANN is used to build predictive models, the advantage of artificial neural network over conventional programming lies in their ability to solve problems that do not have an algorith mic solution or the available solution is too complex to be found. Neural networks are well suited to tackle problems that people are good at solving, like prediction and pattern recognition.

Hence, the growing need and lack of a veritable cum reliable tool for prediction of the stock market serve as impetus for this thesis. Therefore, this could prove to be a vital tool for investment decision making since it is expected to assist investors in making better and quality decisions and managing their stocks much more profitably. However, the difficulty in identifying good raw data, pre-processing this data, training an artificial neural network and repeating this process until a good model is developed should not be discounted.

Predicting stock market data has proven to be difficult due to erratic and unpredictable market 
behavior. The stock market is characterized by so many fluctuations such as political (or industrial) climate, government policies, economic indicators, etc and mainly as a consequence of the close to random-walk behavior of a stock time series. All these have made it pretty arduous to predict accurate future trends in the Stock Market specifically price of stocks.

In Nigeria, there has been almost no research that deals with prediction of Nigerian stock market value. In this paper, we present the result of Nigerian Stock Exchange (NSE) market index values prediction using ANN.

\section{Artificial Neural Network: An Overview}

Artificial Neural Network (ANN) grew out of research in Artificial Intelligence specifically attempts to mimic the fault-tolerance and capacity to learn of biological neural systems by modelling the low-level structure of the brain [3]. According to Anderson and Rosenfeld in 1989, an artificial neural network is a relatively crude electronic model based on the neural structure of the brain [4].

The brain basically learns from experience. It is natural proof that some problems that are beyond the scope of current computers are indeed solvable by small energy efficient packages. This brain modelling also promises a less technical way to develop machine solutions. This approach to computing also provides a more graceful degradation during system overload than its more traditional counterparts [1]. This technique does not require the previous development of algorithms or rule sets to analyze data. This can significantly minimize the software-development work needed for a given application. In most cases, the neural network is previously submitted to a training step using known data; then the methodology necessary to perform the required data processing is extracted. Thus, a neural network is able to identify the required relationships from real data, avoiding the previous development of any model [5].

ANN is analogous to the human brain. The exact working of the human brain is still a mystery. Yet, some aspects of this amazing processor are known. In particular, the most basic element of the human brain is a specific type of cell which, unlike the rest of the body, does not appear to regenerate. Because this type of cell is the only part of the body that isn't slowly replaced, it is assumed that these cells are what provide us with our abilities to remember, think, and apply previous experiences to our every action. These cells, all 100 billion of them, are known as neurons. Each of these neurons can connect with up to 200,000 other neurons, although 1,000 to 10,000 are typical. A typical biological neuron is as shown in figure 1 .

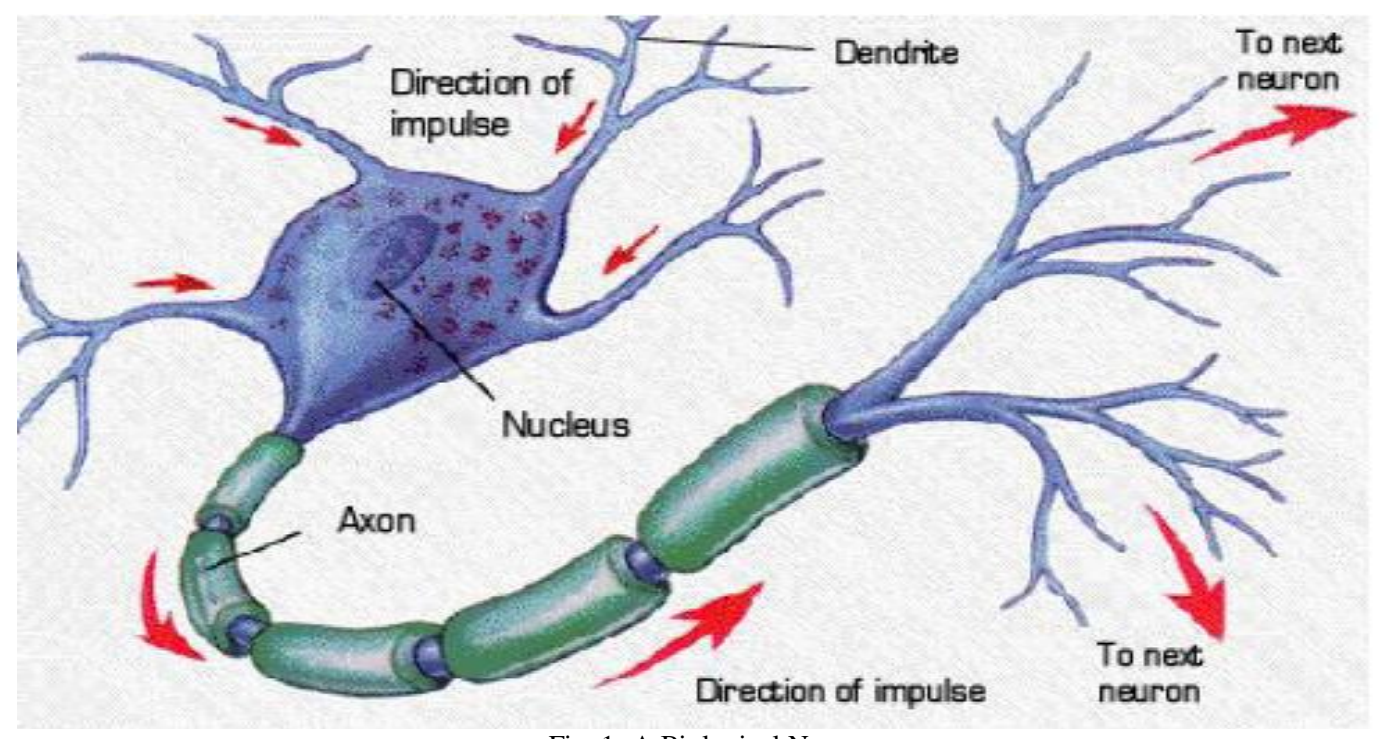

Fig. 1: A Biological Neuron

Basically, the general artificial neuron model has five components, which consists of the following:

(a) Inputs $\left(\mathrm{x}_{\mathrm{i}}\right)$ : The input layer of an ANN typically functions as a buffer for the inputs, transferring the data to the next layer. Pre-processing the inputs may be required as ANNs deal only with numeric data. This may involve scaling the input data and converting or encoding the input data to a numerical form that can be used by the ANN. (b) Weighting schemes (i.e. weight initialization and weight learning algorithms): The first thing an artificial neuron does is to compute the weighted sum of its inputs (i.e., the inner product between the input pattern and the connection strengths). The weights are real-valued numbers that determine the contribution of each input. The goal of neural network training algorithms is to determine the "best" possible set of weight values for the problem under consideration. Finding the 
optimal set is often a trade-off between computation time, minimizing the network error, and maintaining the network's ability to generalize. The back-propagation algorithm is by far the most popular learning algorith $\mathrm{m}$ for multilayer networks and was used in this work.

(c) Threshold (u): The threshold is a real number that is subtracted from the weighted sum of the input values. Sometimes the threshold is called a bias value. In this case, the real number is added to the weighted sum. For simplicity, the threshold can be regarded as another input / weight pair, where $\mathrm{w}_{0}=$ $\mathrm{u}$ and $\mathrm{x}_{0}=-1$.

(d) Transfer (Activation) Functions: The transfer or activation function is a function that determines the output from a summation of the weighted inputs of a neuron. The transfer functions for neurons in the hidden layer are often nonlinear and they provide the nonlinearities for the network. The activation function for the orig inal McCulloch-Pitts neuron was the unit step function. According to Pesaran and Timmermann in 1999, the artificial neuron model has since been expanded to include other functions such as the sigmoid (log istic), piecewise linear, and Gaussian [6].

(e) The Neuron Output (y): The artificial neuron computes its output according to the equation shown below. This is the result of applying the activation function to the weighted sum of its inputs, less the threshold. This value can be discrete or real depending on the activation function used.

$$
y=f\left(\sum_{i=1}^{n} w_{i} x_{i}-u\right)=f\left(\sum_{i=0}^{n} w_{i} x_{i}\right)
$$

Once the output has been calculated, it can be passed to another neuron (or group of neurons) or sampled by the external environment. The interpretation of the neuron output depends upon the problem under consideration. A simplified artificial neuron is as shown in figure 2.

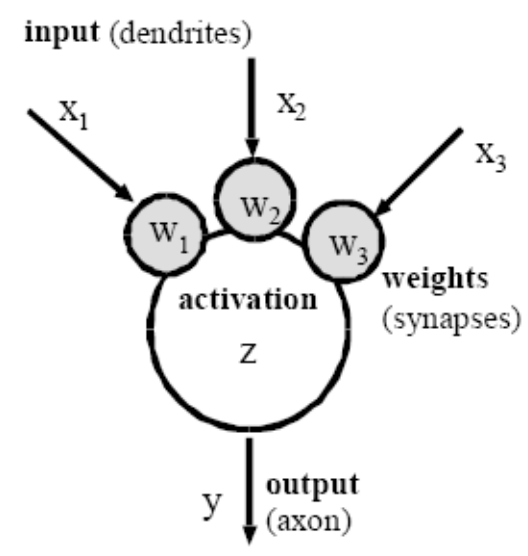

Fig. 2: A Simplified Artificial Neuron

\section{Learning Paradigms in ANN}

Learning is the weight modification process of an ANN in response to external input. This is done with the aim of minimizing the error at the output of the network. The variation of the weights is controlled by the learning of the neural network. In the learning (or training) phase, the learning patterns are presented to the network in many cycles. If the learning phase is very long, the network tends to be over adapted to the learning patterns. All input values not included in the learning will lead to big errors. To avoid over adaptation, the neural network is validated with different patterns, not used for the learning. If the error of the validation patterns increases, the network tends to be over adapted and the training should be stopped.

There are basically two types of learning in ANN, and these include:

1. Supervised learning: It is by far the most common type of learning in ANNs. It requires many samples to serve as exemplars. Each sample of this training set contains input values with corresponding desired output values (also called target values). The network would then attempt to compute the desired output from the set of given inputs of each sample by minimizing the error of the model output to the desired output. It attempts to do this by continuously adjusting the weights of its connection through an iterative learning process called training. As mentioned earlier on, the most common learning algorithm for training the network is the back-propagation algorithm; this would be readily employed in this research work. Tasks that fall within the paradig $\mathrm{m}$ of supervised learning are pattern recognition (also known as classification) and regression (also known as function approximation). The supervised learning paradigm is also applicable to sequential data (e.g., for speech and gesture recognition).

2. Unsupervised learning: It is sometimes called selfsupervised learning and requires no explicit output values for training. Each of the sample inputs to the network is assumed to belong to a distinct class. Thus, the process of training consists of letting the network uncover these classes. It is not as popular as supervised learning. Tasks that fall within the paradigm of unsupervised learning are in general estimation problems; the applications include clustering, the estimation of statistical distributions, compression and filtering.

In $\mathrm{ANN}$, there are many learning algorithms (rules) in common used, in this paper we discuss the most popular two: the Delta rule and its generalization, and the Back propagation algorithm .

\subsection{Delta Rule/ Least Mean Squares (LMS)}

The Least Mean Square (LMS) algorithm was first proposed by Widrow and Hoff in 1960 when they 
introduced the ADALINE (Adaptive Linear Element), an ANN model that was similar to the perceptron model except that it only has a single output neuron and the output activation is a discrete bipolar function that produces a value of 1 or -1 . The LMS algorithm was superior to Rosenblatt's perceptron learning algorithm in terms of speed but it also could not be used on networks with hidden layers.

Most literature claims the Delta Rule and the LMS Rule is one and the same [5] [7].

They are, in terms of the weight change, $\Delta \mathrm{w}_{\mathrm{ij}}$, the formula is given as:

$$
\Delta \mathrm{w}_{\mathrm{ij}}=\eta \delta_{\mathrm{j}} \mathrm{x}_{\mathrm{i}}
$$

Where $\eta \quad$ is the learning rate $(0<\eta<1)$ and $\delta \mathrm{j}$ is the error at neuron $\mathrm{j}$. This rule can also be rewritten as:

$$
\Delta \mathrm{w}=-\eta\left(\mathrm{t}_{\mathrm{p}}-\mathrm{x}_{\mathrm{p}} \mathrm{w}^{\mathrm{p}}\right) \mathrm{x}_{\mathrm{p}}
$$

\subsection{Back-Propagation (BP) / Generalized Delta Rule}

The back-propagation (BP) algorithm is a generalization of the Delta rule that works for networks with hidden layers. It is by far the most popular and most widely used learning algorithm by ANN researchers[1]. Its popularity is due to its simplicity in design and implementation. The idea is to train a network by propagating the output errors backward through the layers. The errors serve to evaluate the derivatives of the error function with respect to the weights, which can then be adjusted. It involves a twostage learning process using two passes: a forward pass and a backward pass. In the forward pass, the output $\left(\mathrm{O}_{\mathrm{j}}\right)$ is computed from set of input patterns, $\mathrm{X}_{\mathrm{i}}$. This can be expressed mathematically as:

$$
\mathrm{Oj}=\mathrm{f}\left(\sum \mathrm{w}_{\mathrm{ij}} \mathrm{x}_{\mathrm{i}}, \theta_{\mathrm{j}}\right)
$$

Where $\mathrm{f}$ is a nonlinear transfer function, e.g. sigmoid function, $\theta_{\mathrm{j}}$ is the threshold value for neuron $\mathrm{j}, \mathrm{x}_{\mathrm{i}}$ is the input from neuron $\mathrm{i}$ and $\mathrm{w}_{\mathrm{ij}}$ represents the weight associated with the connection from neuron $i$ to neuron $j$.

After the output of the network has been computed, the learning algorithm is then applied from the output neurons back through the network, adjusting all the necessary weights on the connections in turn using equation (2) above. There are many other variations to the BP algorithm, but by far, BP still proves to be the most popular and is implemented in almost all commercial ANN software packages.

\section{Related Works}

The birth of artificial neural network has aroused interests in their applications in various areas, and one of them is the stock market prediction. ANN has been used in stock market prediction by many researchers. In Tokyo researchers have used ANN to predict Tokyo Stock Exchange index [8] and buying and selling signals [9]. Singapore stock exchange market direction was also predicted using ANN [10]. Harvey in 1995, in his attempt to predict stock market, he had created a three multi-layer, back propagation artificial neural networks using macroeconomic indicators as inputs. His data set consisted of values for six variables over a 150 months period extending from June 1982 to December 1994. His networks were trained on the first 75 months of the data set, and then tested on the remaining 75 months [11].

Apart from stock market prediction, ANN was also used in prediction of financial issues. In Turkey, it was used to predict financial failure [12]. Sitte and Sitte in 2000, reports on financial time series prediction using neural network. The S\&P 500 index data used in their studies covers 22 years, from 1973 to 1994. They did two (2) sets of experiments. In both experiments, the networks were trained to predict one day ahead. For the first set of experiments the motive was to find the best time delay feed forward network predictor. It was done in a systematic short-term corre lation experiment where the past data input to the predictor ranged from one day to a month. This range was the window size. For the second group of experiment, they have tested a set of networks by systematically changing the window size and number of nodes in the hidden layer [13]. In the literature, there is no research that predicts the Nigerian stock market index which is the focus of this paper.

\section{Methods}

Stock Market data from the first quarter of 2003 to the first quarter of 2006 of four selected banks were collected. They banks are Union Bank of Nigeria PLC, Zenith Bank of Nigeria PLC, First Bank of Nigeria PLC, Guarantee Trust Bank of Nigeria PLC. The banks were used because they are most active stock in Nigerian stock exchange and the four banks used are the leading banks.

The collected data (historical stock market price) was analysed so that there are no leaking values (redundant stock data owing to technical suspension of the stock by the regulatory authorities - Nigerian Stock Exchange). In other words, the input data has to be free of noise, removal of those "wrong" values will increase the capabilities of the ANN model to be better trained, to better capture the underlying regularities in the time series data, and consequently to better predict the future values. The values was rescaled, usually they are so scaled so that the mean of the time series becomes zero (0) and variance one (1). This is accomplished through the use of the pre-processing and post processing functions available in MATLAB 6.5 Software. This has to be done to assure that the training function can be used and will be calculating the proper results.

Once the neural network was structured the training, or learning, begins. The neural network then processes the inputs and compares its resulting outputs against the 
desired outputs. Errors are then propagated back through the system, causing the system to adjust the weights which control the network. This process occurs over and over as the weights are continually tweaked. The set of data which enables the training is called the 'training set'. During the training of the network the same set of data is processed many times as the connection weights are ever refined. To monitor if the ANN predictive model is simply memorizing its data in some non-significant way, there was a need to hold back a set of data to be used to test the model after it has undergone its training. Memorization is avoided by not having too many proces sing elements-neurons.

The simulation was done using MATLAB (Matrix laboratory). MATLAB offers a unique simulation and prototyping environment. It is both concise and descriptive allowing one to model complex and dynamic system like the stock market with small sections of easy-to-flow-code. MATLAB offers an array of tools for simulation and modelling techniques. Application specific tool boxes, such as optimization, control system, neural network; etc allows one to quickly build simulations and models for applications across a range of disciplines with limited coding from scratch. To visualize the simulation results as they calculate or for post-processing, built-in animation functions with graphics allow one to view model behaviours for analysis, testing and debugging, and presentation purposes. All these features and more make MATLAB an indispensable tool for use in this work.

\section{Result and Discussion}

This was accomplished using the post training analysis. This provided a measure through which the performance of the stock predictive model was measured to some extent by investigating the network response and the corresponding targets. The post training analysis as well as the mathematical models generated from it for the four bank stocks covered in this paper are given below in the following figures.

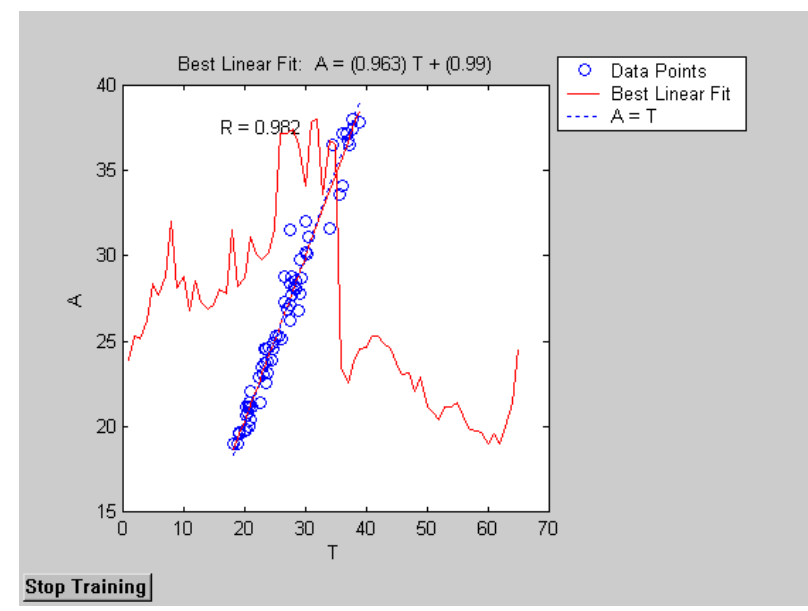

Fig. 3: Post Training Analysis for Union Bank's Stock Price

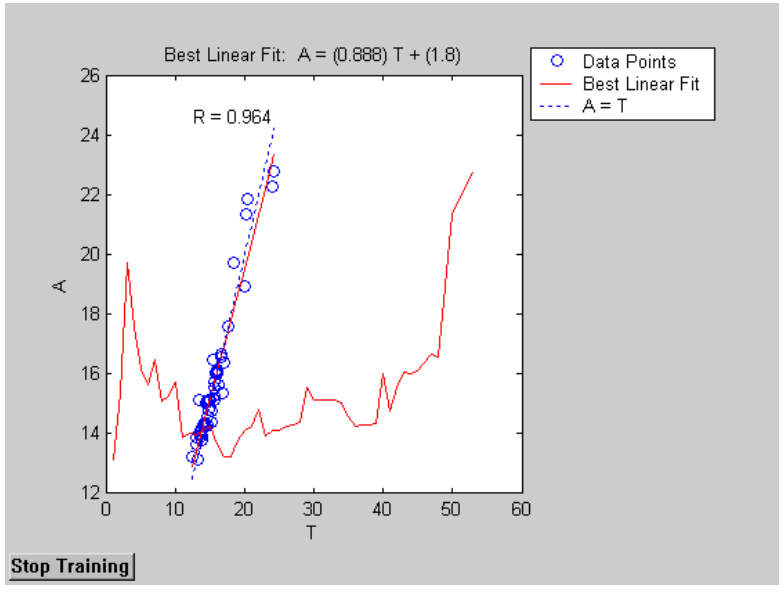

Fig. 4: Post Training Analysis for Zenith Bank's Stock Price

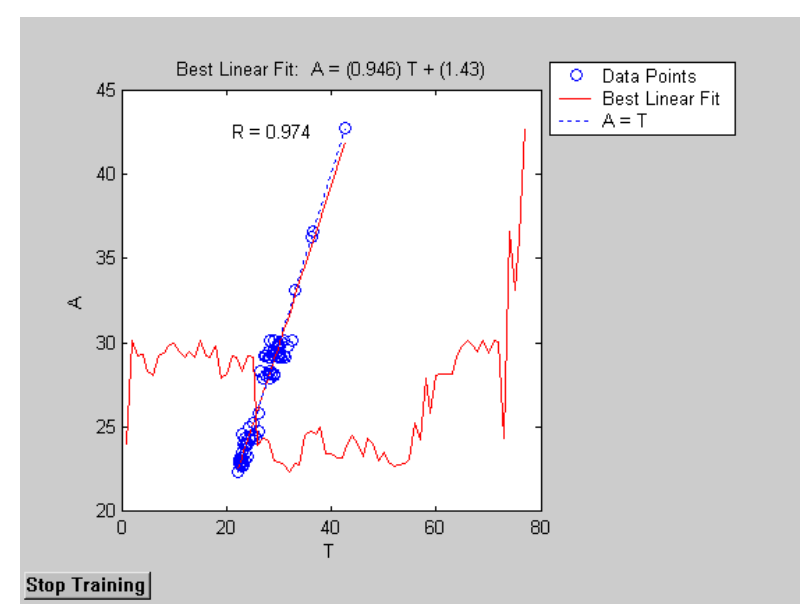

Fig. 5: Post Training Analysis for First Bank's Stock Price

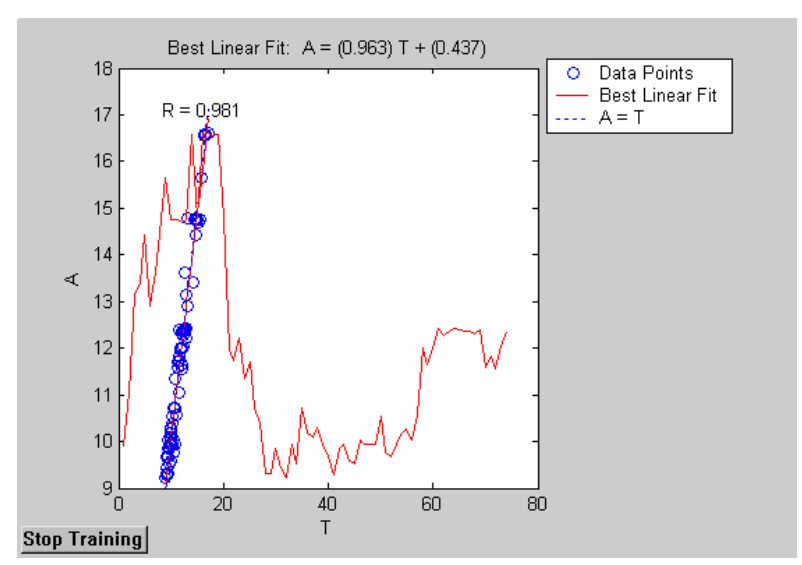

Fig. 6: Post Training Analysis for GT Bank's Stock Price

From the figures 3 to 6 above, the post training analys is returned three parameters. The first two, $m$ and $\mathrm{b}$, correspond to the slope and the $\mathrm{y}$-intercept of the best linear regression relating targets to network outputs. If we had a perfect fit (outputs exactly equal to targets), the slope would be 1 , and the $y$-intercept would be 0 . In this research work, it is obvious that the numbers are very close. The third variable returned by the post training analysis is the correlation coefficient (R-value) between the outputs and targets. 
This is a measure of how well the variation in the output is explained by the targets. If this number is equal to 1 , then there is perfect correlation between targets and outputs. In this research work, the number is very close to one (1) for all the bank stocks covered, which indicates that the ANN model is a good fit. The network outputs are plotted versus the targets as open circles. The best linear fit is indicated by a dashed line. The perfect fit (output equal to targets) is indicated by the solid line. In this research work, it is difficult to distinguish the best linear fit line from the perfect fit line, because the fit is so good owing to the input data, training data and the training procedures adopted.

After the post training analysis, the model was deployed in the real environ ment - stock data (not in the training set) was fed to the neural network and prediction was made based on the ANN model. The dynamic mathematical models generated from the post training analysis for GT Bank's stock, First Bank's stock, Union Bank's stock and Zenith bank's stock are as shown in the following equations.

$$
\begin{aligned}
& \mathrm{A}=0.963 \mathrm{~T}+0.437 \\
& \mathrm{~A}=0.946 \mathrm{~T}+1.43 \\
& \mathrm{~A}=0.963 \mathrm{~T}+0.99 \\
& \mathrm{~A}=0.888 \mathrm{~T}+1.8
\end{aligned}
$$

The letter ' $A$ ' is the actual stock price to be predicted while ' $T$ ' is used as the training data (today's stock price) which will be used for making prediction.

The accuracy of the prediction was measured using the concept of relative error and the results were quite outstanding. Its ability to predict the ups and downs of stock price was much more amazing to me, though, it did not achieve 100 percent level of accuracy. The model had a mean square error of 0.9365 (First Bank stock), 0.9446(Union Stock), 0.8846(Zenith Stock), and 0.9500 (GT Stock).

\section{Conclusion}

Perhaps the greatest advantage of ANNs is their ability to be used as an arbitrary function approximation mechanis $m$ which 'learns' from observed data. The implementation of the artificial neural network ran the same training data multiple times, to allow the weights more updates for better convergence. Each run of the training data is referred to as epoch. By varying the number of epochs, we can easily vary the training data size (effectively running the training data multiple times.) Also, as a rough cross-validation mechanism, at the end of each training run, the end of each epoch, the network is tested, and updated against the test set.

The result of this work has shown really that artificial neural network when trained can be used to predict future stock prices. With the correct implementation
ANNs can be used naturally in predicting stock market trends. Under "normal" conditions, in most cases, a good neural network will outperform most other predictive models and be a very worthwhile and potentially profitable aid to investors However, the difficulty in identifying good raw data, pre-processing this data, training a network and repeating this process until a good model is developed should not be discounted. Regardless of ANNs outputs, a user should always do their due diligence prior to investing in any investment. As good as ANN can be, one must understand it is not a crystal ball to the future since it is unlikely a neural network will ever be the perfect prediction device that is desired because the factors in a large dynamic system, like the stock market, are too complex to be understood for a long time. No human or computer can perfectly predict the volatile stock market!

\section{Acknowledgment}

The authors acknowledged the referees, Iy inoluwa, Iyanuoluwa, Titilope and Hezekiah Oluwasanmi library, Obafemi Awolowo University, Nigeria.

\section{References}

[1] Bishop, C.M. Neural Networks for Pattern Recognition, Oxford: Oxford University Press, 1995

[2] Okobiah, E. Monetary Studies (Theories and Policies), Delta State University, Lagos Centre., 2000.

[3] Pesaran, M.H. and Timermann, M. A Recursive Modelling Approach to Predicting Stock Returns, University of Cambridge Press, Cambridge, 1999.

[4] Anderson, M. and Rosenfeld, E. Neural Networks - Theory and Architecture, Prentice Hall, London, 1989.

[5] Chung, K.K. Financial Forecasting using Neural Networks or Machine Learning Techniques, University of Queensland, Queensland, 2001 \{unpublished BSc. Thesis ]

[6] Pesaran, M.H. and Timermann, M. A Recursive Modelling Approach to Predicting Stock Returns, University of Cambridge Press, Cambridge, 1999.

[7] Carling, A. Introducing Neural Networks, Sig ma Press, England, 1992

[8] Kimoto T., Asakawa K., Yodaa M., Takeoka M. Stock Market prediction System with modular neural network, in proceedings of the International Joint Conference on Neural Network, 1990, pp 1-6.

[9] Mizuno H., Kosaka M., Yajima H., Komoda N. Application of Neural Network to Technical Analysis of Stock Market Prediction, Studies in 
informatics and Control, 1998, Vo17 No 3 pp 111 120.

[10] Phua P., Ming D. Lin W., Neural Network With genetic Algorithms for Stock prediction, $5^{\text {th }}$ Conference of the Association of Asian Pacific Operations Research Societies, 5-7 July 2000, Singapore

[11] Harvey, B. Stock Market Prediction using Artificial Neural Networks: A macroeconomic Approach, University of Queensland, 1995.

[12] Yildiz B., Use of Artificial Neural Network in prediction of Financial Failures Journal of IMKB, 2001, Vol 5 No 17.

[13] Sitte, R. and Sitte, J. 'Analysis of Predictive Ability of Time Delay Neural Networks Applied to the S\&P 500 Time Series, Proceedings of the IEEE Transactions on Systems, Man and Cybernetics Part C: Applications and Reviews', 2000, Vol. 30, No 4, pages 568 - 572.

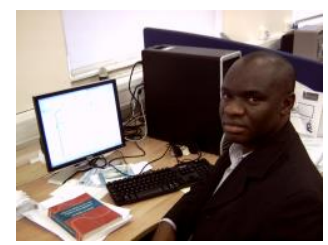

Adebayo Peter Idowu: Male, Nigeria, Lecturer at the Obafemi A wolowo University, Nigeria. His research interests include Software Engineering, Geographical Information System, Database Design, and Health Informatics.

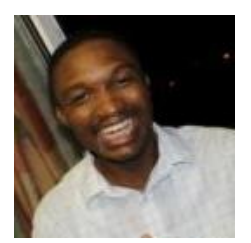

Chris Osakwe: Male, Nigeria, His research interests include Software Engineering, Database Design, and Simulation.

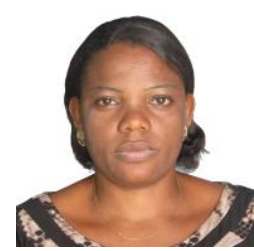

\begin{abstract}
Aderonke Anthonia Kayode: Female, Nigeria, Her research interests include Software Engineering, information Retrieval, and Health Informatics.
\end{abstract}

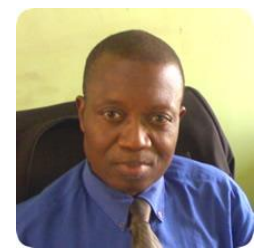

Emmauel Rotimi Adagunodo: Male, Nigeria, Professor of Computer Science \& Engineering in Obafemi Awolowo University, Nigeria. His research interests include Software Engineering, and
How to cite this paper: Peter Adebayo Idowu, Chris Osakwe, Aderonke Anthonia Kayode, Emmanuel Rotimi Adagunodo,"Prediction of Stock Market in Nigeria Using Artificial Neural Network", International Journal of Intelligent Systems and Applications(IJISA), vol.4, no.11, pp.68-74, 2012. DOI: 10.5815/ijisa.2012.11.08

operating system. 